\title{
Absorption et régénération de l'azote dans le système brassé de la Manche : productions nouvelle et régénérée
}

\author{
Jean-François MAGUER, Stéphane L'HELguen, Christian MADEC, Pierre LE CORRE \\ Laboratoire de chimie marine, UPR 9042 CNRS Roscoff \\ et université de Bretagne occidentale, place Nicolas-Copernic, Technopôle Brest-Iroise, 29280 Plouzané, France
}

(Révisé le 11 mai 1998, accepté le 14 mai 1998)

\begin{abstract}
Uptake and regeneration of nitrogen in the well-mixed waters of the English Channel: new and regenerated productions. Nitrate and ammonium uptake rates were measured in spring and summer in deep and shallow wellmixed waters of the English Channel during different cruises between 1986 and 1994. In the deep waters, nitrate uptake was relatively low during phytoplankton development and ammonium uptake represented more than $70 \%$ of the total uptake irrespective of the season. In the shallow waters, nitrate uptake during spring phytoplankton growth was high and represented about $75 \%$ of the total uptake. Ammonium uptake became substantial towards the end of spring and summer. The high contribution of ammonium to the nitrogenous nutrition of phytoplankton over the wholc of the well-mixed waters is related to a high rate of recycling of nitrogen in the water column. Ammonium regeneration by microheterotrophs can satisfy between $62 \%$ and the totality of the phytoplankton nitrogen requirements. The high primary production in decp well-mixed waters is not supported by allochthonous nitrogen supply but by an intense in situ regeneration of nitrogen. The situation is similar in shallow well-mixed waters, except during the spring bloom. $\odot$ Elsevier, Paris
\end{abstract}

\section{nitrogen / uptake / regeneration / new and regenerated productions / English Channel}

Résumé - L'absorption du nitrate et de l'ammonium a été étudiée au printemps et en été dans les systèmes brassés profond et peu profond de la Manche. Les travaux ont été réalisés lors de différentes campagnes à la mer menées entre 1986 et 1994. Dans le système brassé profond, le nitrate est peu utilisé durant la période de développement du phytoplancton et l'absorption de l'ammonium représente, quel que soit le secteur, plus de $70 \%$ de l'absorption totale. Dans le système brassé peu profond, l'absorption du nitrate s'avère élevée en période de floraison du phytoplancton. Elle représente alors $75 \%$ de l'absorption totale. La contribution de l'ammonium à la nutrition azotée du phytoplancton est marquée à la fin de la floraison printanière et en période estivale. Cette contribution élevée de l'ammonium est liée, dans l'ensemble du système brassé, à un recyclage rapide de l'azote dans la colonne d'eau. La régénération de l'ammonium par les microhétérotrophes permet de satisfaire entre $62 \%$ et la totalité des besoins du phytoplancton. Les valeurs élevées de production primaire dans le système brassé profond ne résultent pas d'apports nouveaux en azote mais correspondent à de fortes productions régénérées. En dehors de la floraison printanière du phytoplancton, la situation est similaire dans le système brassé peu profond. (C) Elsevier, Paris

azote / absorption / régénération / productions nouvelle et régénérée / Manche

\section{INTRODUCTION}

La Manche est un exemple type de mer côtière à régime macrotidal. Les courants de marée y sont si intenses qu'ils empêchent l'établissement d'une stratification en surface lors du réchauffement estival : dans la majeure partie de la Manche, la colonne d'eau demeure alors homogène toute l'année. Les cycles de production primaire et de sels nutritifs y sont tout à fait particuliers. La production primaire est caractérisée, lorsque l'épaisseur 
de la colonne d'eau est importante et nettement supérieure à celle de la zone euphotique (système brassé profond), par un large maximum qui s'étend durant toute la période estivale $[2,12,38]$. Le nitrate n'est pas épuisé en été et l'absorption de l'azote n'est pas limitée par sa concentration [25]. Parallèlement au développement du phytoplancton, les teneurs en ammonium augmentent dans la colonne d'eau pour atteindre un maximum en été. Des productions primaires élevées $\left(250-350 \mathrm{~g} \mathrm{~m}^{-2} \mathrm{a}^{-1}\right.$ de $C,[2,38]$ ) caractérisent ce système. Il a été démontré que cette forte production ne résulte pas d'apports anthropiques en éléments nutritifs, mais correspond à un recyclage rapide de l'azote dans la colonne d'eau [24]. Dans le cas particulier de la frange côtière, où l'épaisseur de la colonne d'eau brassée est fajble et où la couche euphotique atteint le fond en été (système brassé peu profond), il n'y a pas de maximum de production estival. Le cycle de production dans ce système présente un maximum marqué au printemps [28]. Le développement phytoplanctonique engendre un épuisement du nitrate dont l'absorption est limitée de la fin du printemps à la fin de l'été. Néanmoins, le système brassé peu profond présente aussi une production primaire principalement de nature régénérée [29]. L'ensemble de ces résultats a été établi à deux stations côtières situées en Manche occidentale, au voisinage de Roscoff. Les flux d'absorption et de régénération de l'azote ont été mesurés dans les différents secteurs de la Manche lors du Programme national d'océanographie côtière (PNOC) et au cours de campagnes antérieures au PNOC (tableau I). Dans cet article. nous nous proposons de présenter ces nouveaux résultats et de les comparer avec ceux obtenus dans le secteur de Roscoff. L'objectif est de généraliser les résultats acquis à l'ensemble de la Manche.

\section{MATÉRIEL ET MÉTHODES}

\subsection{Position des stations et techniques de prélèvements}

Neufs stations, réparties en Manche occidentale et en Manche orientale, ont été étudiées (figure 1), les stations $1,3,4,5$ et 6 au printemps, les stations $\mathbf{M}_{2}, \mathbf{A}_{2}, \mathrm{~A}_{3}$ et $\mathrm{A}$ en période estivale (tableau $I$ ). La ligne de séparation entre les systèmes profond (stations $1,3, M_{2}, A_{2}$ et $A_{3}$ ) et peu profond (stations 4, 5, 6 et $\mathrm{A}$ ) correspond approximativement à l'isobathe $30 \mathrm{~m}$, limite d'extension des eaux côtières épuisées en nitrate en été.

Les taux d'absorption de l'ammonium et du nitrate et les taux de régénération de l'ammonium ont été mesurés à chaque station, à l'exception de la station $\mathrm{A}$, où seuls les taux d'absorption ont été déterminés. Les prélèvements ont été effectués aux environs de $9 \mathrm{~h}$ du matin à l'aide de bouteilles Niskin de $30 \mathrm{~L}$. Cinq niveaux ont été habituellement échantillonnés dans la zone euphotique et un dans la couche sous-jacente. Les échantillons ont été préfiltrés à $200 \mu \mathrm{m}$. Les taux d'absorption et de régénération correspondent donc à l'activité du microplancton.

\subsection{Sels nutritifs, azote particulaire et chlorophylle $a$}

Les concentrations en nitrate ont été déterminées par autoanalyseur Technicon type AAll suivant la procédure décrite par Tréguer et Le Corre [37]. Celles de l'ammonium ont été mesurées en triplicat par la méthode manuelle de Koroleff [19]. Les précisions en nitrate et ammonium sont respectivement de 0,05 et $0,02 \mu \mathrm{mol} \mathrm{L}^{-1}$. L'azote organique particulaire (NOP) a été collecté sur des filtres $\mathrm{GF} / \mathrm{F}$ (diamètre $=47 \mathrm{~mm}$ ) prétraités à $450^{\circ} \mathrm{C}$ durant $4 \mathrm{~h}$; les mesures ont été réalisées sur analyseur

Tableau I. Campagnes, coordonnées géographiques et profondeurs des stations aux points de prélèvements.

Table 1. Cruises, geographical positions and depths at sampling stations.

\begin{tabular}{lcccc}
\hline Stations & $\mathbf{Z}(\mathbf{m})$ & Latitude nord & Longitude & Campagnes \\
\hline Station $\mathrm{M}_{2}$ & 75 & $48^{\circ} 31.10^{\circ}$ & $4^{\circ} 56.10^{\prime} \mathrm{W}$ & Iroise, juillet 1986 \\
Station $\mathrm{A}_{2}$ & 91 & $48^{\circ} 48.20^{\prime}$ & $4^{\circ} 21.35^{\prime} \mathrm{W}$ & Azonix, juillet 1988 \\
Station $\mathrm{A}_{3}$ & 73 & $48^{\prime} 47.00^{\prime}$ & $4^{\circ} 07.00^{\prime} \mathrm{W}$ & - \\
Station 1 & 80 & $48^{\circ} 51,20^{\prime}$ & $3^{\circ} 53.20^{\prime} \mathrm{W}$ & CPB Manche I (PNOC), mai-juin 1993 \\
Station 3 & 40 & $48^{\circ} 49.80^{\prime}$ & $2^{\circ} 39.80^{\prime} \mathrm{W}$ & - \\
Station 4 & 25 & $49^{\circ} 37.60^{\prime}$ & $0^{\circ} 12.57^{\prime} \mathrm{W}$ & - \\
Station 5 & 30 & $50^{\circ} 54.50^{\prime}$ & $1^{\circ} 34.00^{\prime} \mathrm{E}$ & - \\
Station 6 & 23 & $50^{\circ} 48.00^{\prime}$ & $1^{\circ} 19.50^{\prime} \mathrm{E}$ & Nutriseine II (PNOC), juin 1994 \\
Station A & 38 & $49^{\circ} 45.15^{\prime}$ & $0^{\circ} 00.25^{\prime} \mathrm{W}$ & \\
\hline
\end{tabular}




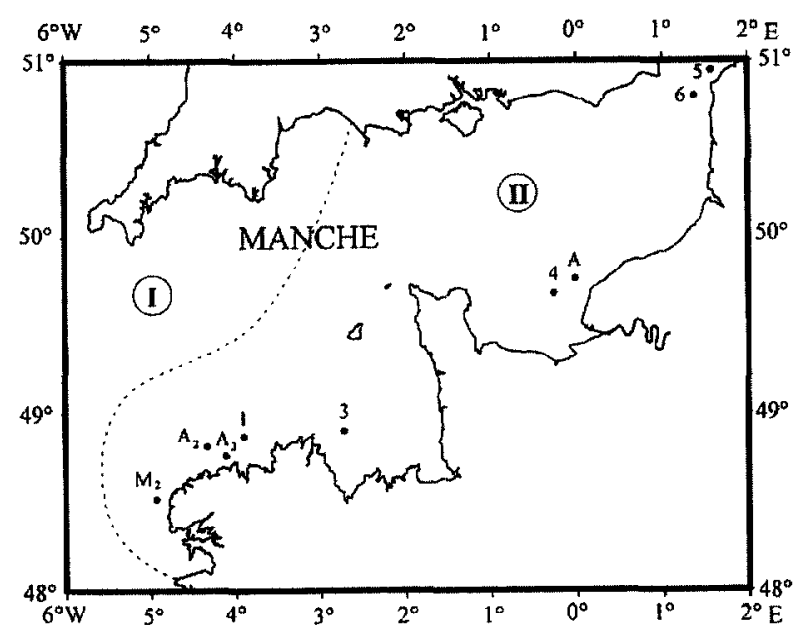

Figure 1. Emplacement des stations de prélèvements. (I) le système stratifié, (II) le système brassé, en pointillé : le front d'Ouessant.

Figure 1. Position of sampling stations. (I) stratified waters (II) well-mixed waters, dotted line: the Ushant front.

CHN Perkin Elmer (modèle 240) avec une précision de $0,1 \mu \mathrm{mol} \mathrm{L} \mathrm{L}^{-1}$. Les teneurs en chlorophylle $a$ ont été obtenues par la méthode fluorimétrique de Yentch et Menzel [39] avec une précision de $\pm 0,05 \mu \mathrm{g} \mathrm{L}^{-1}$.

\subsection{Absorption et régénération de l'azote}

Les taux d'absorption du nitrate et de l'ammonium ont été obtenus en suivant l'incorporation du traceur ${ }^{15} \mathrm{~N}$ dans la matière particulaire [8], les taux de régénération de l'ammonium par les microhétérotrophes en utilisant la technique de dilution isotopique [14]. Les échantillons d'eau de mer ont été inoculés avec des composés marqués $\left(\mathrm{Na}^{15} \mathrm{NO}_{3}, 99 \%\right.$ atome ${ }^{15} \mathrm{~N}$ et ${ }^{15} \mathrm{NH}_{4} \mathrm{Cl}, 99 \%$ atome ${ }^{15} \mathrm{~N}, \mathrm{CEA}$, France) à une concentration voisine de $10 \%$ du nitrate ou de l'ammonium initialement présent dans l'eau de mer. Lorsque les concentrations initiales étaient proches de la limite de détection, $0,05 \mu \mathrm{mol} \mathrm{L}^{-1}$ de nitrate ou d'ammonium a été additionnée. L'enrichissement initial en ${ }^{15} \mathrm{~N}$ dans la matière particulaire et dans la fraction dissoute a été déterminé. Les échantillons ont été incubés in situ simulé durant $4 \mathrm{~h}(2 \mathrm{~h}$ de part et d'autre de midi solaire) dans des flacons en polycarbonate de 2,5 L. Au cours de la campagne CPB Manche I, des incubations ont été également réalisées la nuit afin d'examiner les variations nycthémérales des différents flux. À la fin des incubations, les échantillons ont été filtrés sur des filtres GГ/T. Les filtres ont été séchés puis conservés en présence d'un déshydratant jusqu'aux analyses isotopiques. Lors des mesures de régénération de l'ammonium, les filtrats ont aussi été récupérés pour la détermination de l'enrichissement en ${ }^{15} \mathrm{~N}$ de ce composé en phase dissoutc. L'ammonium contenu dans la phase dissoute a été extrait par diffusion en milieu basiquc sclon la procédurc décrite par Kristiansen et Paasche [20]. L'enrichissement en ${ }^{15} \mathrm{~N}$ de la NOP et de l'ammonium extrait de la phase dissoute a été mesuré par spectrométrie d'émission (Sopra, modèle GS1) selon la procédure décrite par Guiraud et Fardeau [13].

Les taux d'absorption du nitrate et de l'ammonium ont été déterminés à partir de l'équation établie par Dugdale et Wilkerson [9] qui prend en compte la concentration en NOP au début de l'incubation. Les équations de Glibert et al. [11] ont été utilisées pour la détermination des laux de régénération de l'ammonium lorsque la concentration en ammonium est constante au cours de l'incubation et celles de Laws [21] lorsqu'elle varie. Les coefficients de variation, obtenus pour des taux de $10 \mathrm{nmol} \mathrm{L}^{-1} \mathrm{~h}^{-1}$, sont respectivement de 4 et $6 \%$ pour la détermination des taux d'absorption et de régénération. À la station A (campagne Nutriscine II), les taux de régénération ont été estimés à partir des variations de la concentration en ammonium entre le début et la fin des incubations, en supposant l'absorption de ce composé comme la seule perte d'azote au cours de l'incubation. Ils ont été déterminés avec une précision de $\pm 10 \mathrm{nmol} \mathrm{L}^{-1} \mathrm{~h}^{-1}$. La dilution isotopique n'ayant pas été prise en compte dans les calculs, les valeurs obtenues correspondent à des valeurs maximales. Connaissant les variations nycthémérales des différents flux, les taux journaliers intégrés sur la colonne d'eau ont été déterminés pour le nitrate et l'ammonium aux stations $1,3,4,5$ et 6. Ils ont été estimés aux stations $M_{2}, A_{2}, A_{3}$ et $A$ en multipliant les taux horaires intégrés sur la colonne d'eau par un facteur de 12,4 pour l'absorption du nitrate, de 16,0 pour celle de l'ammonium et de 18,1 pour la régénération de l'ammonium. Ces facteurs correspondent aux valeurs moyennes obtenues en Manche lors de la campagne CPB Manche I.

\section{RÉSULTATS ET DISCUSSION}

\subsection{Température}

Comme le montre la figure 2, la colonne d'eau est thermiquement homogène au printemps et en été aux stations qui ont fait l'objet des travaux. 


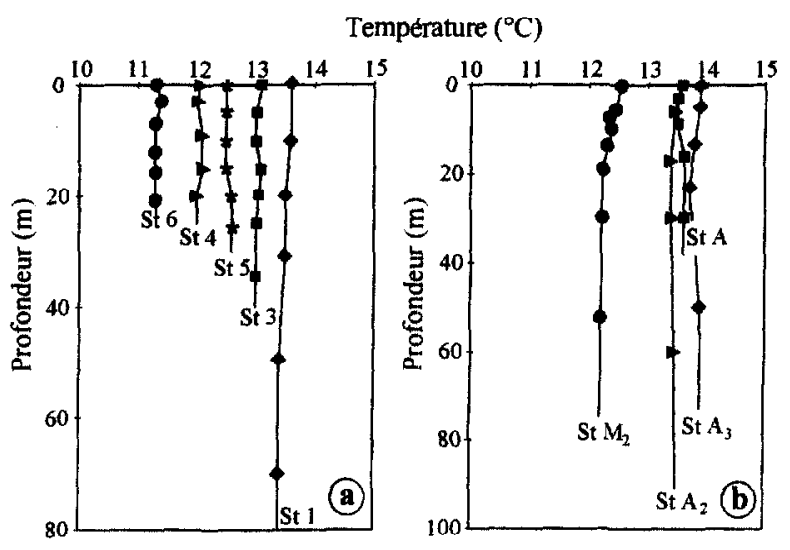

Figure 2. Distribution verticale de la température $\left({ }^{\circ} \mathrm{C}\right)$ aux différentes stations étudiées, (a) au printemps, (b) en été.

Figure 2. Vertical temperature distribution at studied stations (a) in spring, (b) in summer.

\subsection{Sels nutritifs et chlorophylle $a$}

\subsubsection{Système brassé profond}

Au printemps, les teneurs en nitrate dans le système brassé profond sont respectivement de 1 et $2,7 \mu \mathrm{mol} \mathrm{L} \mathrm{L}^{-1}$ aux stations 1 et 3 . Dès cette époque, de fortes concentrations en ammonium sont mises en évidence dans la colonne d'eau (tableau II). Les teneurs en chlorophylle $a$ sont peu élevées $\left(<1 \mu \mathrm{g} \mathrm{L}^{-1}\right)$. En été, le système brassé profond est marqué par des concentrations relativement élevées en nitrate $\left(1,5-2,9 \mu \mathrm{mol} \mathrm{L^{-1 }}\right)$ comparées à celles habituellement observées dans un système stratifié $\left(<0,5 \mu \mathrm{mol} \mathrm{L}^{-1} ;[1]\right)$. Il est aussi caractérisé par la persistance de fortes teneurs en ammonium $(0,56-$ $\left.0,70 \mu \mathrm{mol} \mathrm{L}^{-1}\right)$. La biomasse phytoplanctonique demeure faible à cette époque $\left(<1,3 \mu \mathrm{g} \mathrm{L}^{-1} \mathrm{de}\right.$ chl $\left.a\right)$.

\subsubsection{Système brassé peu profond}

Au printemps, deux situations différentes sont observées dans le système brassé peu profond (tableau II). Les stations 4 et 6 présentent des teneurs élevées en nitrate (respectivement 1,4 et $6,9 \mu \mathrm{mol} \mathrm{L}^{-1}$ ) et significatives en ammonium $\left(0,41\right.$ et $0,13 \mu \mathrm{mol} \mathrm{\textrm {L } ^ { - 1 }}$ respectivement). La biomasse phytoplanctonique est abondante à ces deux stations $\left(5 \mu \mathrm{g} \mathrm{L}^{-1}\right.$ de chl $a$ ). Cette situation correspond à la situation de floraison printanière. La station 5 est marquée par un épuisement du nitrate, des teneurs en ammonium de $0,21 \mu \mathrm{mol} \mathrm{L} \mathrm{L}^{-1}$ et de plus faibles concentrations en chlorophylle $a\left(2,5 \mu \mathrm{g} \mathrm{L}^{-1}\right)$. Ces valeurs sont typiques d'une situation qui suit la floraison du phytoplancton à la fin du printemps et en été. Ces différents résultats montrent qu'il existe, dans le système brassé peu profond, un décalage dans la période de développement printanier du phytoplancton selon les secteurs. Ils sont en accord avec ceux obtenus par Brunet et al. [3] qui mettent en évidence, dans le pas de Calais, un développement plus tardif du phytoplancton dans les eaux de la veine centrale du détroit que dans la frange d'eaux côtières, située entre la baie de la Seine et le cap Gris-Nez [4]. En été, le nitrate à la station A n'est pas épuisé $\left(1,1 \mu \mathrm{mol} \mathrm{L}^{-1}\right)$, les concentrations en ammonium sont relativement fortes

Tableau II. Concentrations en nitrate, ammonium et chlorophylle $a$ dans le système brassé.

Table II. Nitrate, ammonium and chlorophyll $a$ concentrations in the well-mixed waters.

\begin{tabular}{|c|c|c|c|c|}
\hline & Stations & $\left.\begin{array}{c}\mathrm{NO}_{3}^{-} \\
(\mu \mathrm{mol} \mathrm{L} \\
-1\end{array}\right)$ & $\begin{array}{c}\mathrm{NH}_{4}^{+} \\
\left(\mu \mathrm{mol} \mathrm{L}^{-1}\right)\end{array}$ & $\underset{\left(\mu g L^{-1}\right)}{\operatorname{Chl} a}$ \\
\hline \multicolumn{5}{|c|}{ Système brassé profond } \\
\hline Printemps & $\begin{array}{l}\text { Station } 1 \\
\text { Station } 3\end{array}$ & $\begin{array}{l}2,7 \\
1,0\end{array}$ & $\begin{array}{l}1,60 \\
0,66\end{array}$ & $\begin{array}{l}0,2 \\
1,0\end{array}$ \\
\hline Été & $\begin{array}{l}\text { Station } M_{2} \\
\text { Station } A_{2} \\
\text { Station } A_{3}\end{array}$ & $\begin{array}{l}2,9 \\
2,1 \\
1,5\end{array}$ & $\begin{array}{l}0,56 \\
0,70 \\
0,59\end{array}$ & $\begin{array}{l}1,2 \\
0,5 \\
1,2\end{array}$ \\
\hline \multicolumn{5}{|c|}{ Système brassé peu profond } \\
\hline Printemps & $\begin{array}{l}\text { Station } 4 \\
\text { Station } 6 \\
\text { Station } 5 \\
\text { Station A }\end{array}$ & $\begin{array}{r}6,9 \\
1,4 \\
<0,1 \\
1,1\end{array}$ & $\begin{array}{l}0,41 \\
0,13 \\
0,21 \\
0,48\end{array}$ & $\begin{array}{l}5,3 \\
5,6 \\
2,5 \\
4,0\end{array}$ \\
\hline
\end{tabular}


$\left(0,48 \mu \mathrm{mol} \mathrm{L}{ }^{-1}\right)$ et la biomasse phytoplanctonique importante $\left(\sim 4 \mu \mathrm{g} \mathrm{L}^{-1} \mathrm{de} \mathrm{Chl} a\right)$. Cette situation doit être reliée à l'influence des eaux de la Seine, enrichies en nitrate, qui empêche l'épuisement de ce composé et maintient la biomasse phytoplanctonique à un niveau élevé durant l'été.

L'ensemble de ces résultats confirme l'existence dans les systèmes brassés profond et peu profond de cycles particuliers en sels nutritifs et en chlorophylle $a$. Ils montrent aussi que les cycles dans les différents secteurs de la Manche sont similaires. Dans le système brassé peu profond, des décalages apparaissent néanmoins dans la période de développement du phytoplancton.

\subsection{Nutrition azotée du phytoplancton}

\subsubsection{Système brassé profond}

Au printemps, les taux d'absorption du nitrate varient de $<0,1$ à $11,2 \mathrm{nmol} \mathrm{L}^{-1} \mathrm{~h}^{-1}$ dans le système brassé profond (tableau III). Ils sont relativement faibles comparés à ceux habituellement mesurés en régime stratifié lors du développement printanier du phytoplancton $[34,36]$. Des taux d'absorption en ammonium de 1,5 à $12,1 \mathrm{nmol} \mathrm{L}^{-1} \mathrm{~h}^{-1}$ sont mesurés à cette époque. Intégrés sur la colonne d'eau, ils sont trois à quatre fois plus élevés que ceux du nitrate (tableau $V$ ) et représentent plus de $70 \%$ de l'absorption totale. Cette utilisation accrue d'ammonium dès le printemps différencie nettement le système brassé profond des systèmes stratifiés dans lesquels le nitratc peut représenter en période printanière plus de $70 \%$ de l'azote utilisé par le phytoplancton [18, $34,36]$. En période estivale, les taux d'absorption du nitrate varient de $<0,1$ à $13,7 \mathrm{nmol} \mathrm{L}^{-1} \mathrm{~h}^{-1}$ (tableau $\mathrm{III}$ ). Ceux de l'ammonium sont compris entre 1,9 et $39,2 \mathrm{nmol} \mathrm{L}^{-1} \mathrm{~h}^{-1}$; intégrés, ils sont nettement plus élevés que ceux du nitrate (tableau $V$ ) et représentent plus de $80 \%$ de l'absorption totale. Ces résultats confirment ceux déjà obtenus en Manche occidentale au voisinage de

Tableau III. Taux d'absorption du nitrate et de l'ammonium et taux de régénération de l'ammonium dans le système brassé profond.

Table III. Uptake rates of nitrate and ammmonium and regeneration rates of ammonium in the deep well-mixed waters.

\begin{tabular}{|c|c|c|c|c|c|}
\hline & Stations & $\begin{array}{l}\text { Profondeur } \\
\text { (m) }\end{array}$ & $\begin{array}{c}\rho \mathrm{NO}_{3}^{-} \\
(\mathrm{nmol} \mathrm{L} \\
\left.\mathbf{L}^{-1} h^{-1}\right)\end{array}$ & $\begin{array}{c}\rho \mathrm{NH}_{4}^{+} \\
\left(\mathrm{nmol} \mathrm{L} \mathbf{L}^{-1} \mathbf{h}^{-1}\right)\end{array}$ & $\begin{array}{c}\mathrm{RNH}_{4}^{+} \\
\left(\text {nmol } \mathbf{L}^{-1} \mathbf{h}^{-1}\right)\end{array}$ \\
\hline \multirow[t]{2}{*}{ Printemps } & Station 1 & $\begin{array}{r}0 \\
10 \\
20 \\
30 \\
50 \\
70 \\
\end{array}$ & $\begin{array}{r}2,0 \\
2,5 \\
1,9 \\
0,4 \\
<0,1 \\
0,2 \\
\end{array}$ & $\begin{array}{l}5,3 \\
8,1 \\
5,8 \\
3,4 \\
1,7 \\
1,5 \\
\end{array}$ & $\begin{array}{r}10,2 \\
8,8 \\
7,5 \\
10,1 \\
6,7 \\
6,8 \\
\end{array}$ \\
\hline & Station 3 & $\begin{array}{r}0 \\
5 \\
10 \\
15 \\
25 \\
35 \\
\end{array}$ & $\begin{array}{r}9,6 \\
11,2 \\
5,1 \\
3,4 \\
<0,1 \\
<0,1 \\
\end{array}$ & $\begin{array}{r}12,1 \\
9,9 \\
11,8 \\
6,4 \\
1,7 \\
2,0 \\
\end{array}$ & $\begin{array}{r}9,5 \\
8,9 \\
14,4 \\
21,1 \\
20,6 \\
25,7 \\
\end{array}$ \\
\hline \multirow[t]{3}{*}{ Été } & Station $\mathrm{M}_{2}$ & $\begin{array}{r}0 \\
6 \\
8 \\
10 \\
14 \\
19 \\
30 \\
\end{array}$ & $\begin{array}{r}13,7 \\
6,2 \\
10,1 \\
5,8 \\
2,1 \\
1,9 \\
2,2 \\
\end{array}$ & $\begin{array}{r}33,4 \\
25,8 \\
39,2 \\
26,2 \\
13,7 \\
11,1 \\
6,3 \\
\end{array}$ & $\begin{array}{r}26,7 \\
23,0 \\
25,5 \\
21,1 \\
9,9 \\
12,5 \\
4,9 \\
\end{array}$ \\
\hline & Station $A_{2}$ & $\begin{array}{r}0 \\
6 \\
17 \\
30 \\
60 \\
\end{array}$ & $\begin{array}{r}5,4 \\
3,8 \\
1,2 \\
0,5 \\
<0,1 \\
\end{array}$ & $\begin{array}{r}14,6 \\
12,1 \\
9,7 \\
6,3 \\
1,9 \\
\end{array}$ & $\begin{array}{r}11,2 \\
11,6 \\
11,1 \\
4,1 \\
3,6 \\
\end{array}$ \\
\hline & Station $A_{3}$ & $\begin{array}{r}0 \\
5 \\
13 \\
23 \\
50 \\
\end{array}$ & $\begin{array}{l}9,6 \\
7,4 \\
5,6 \\
1,0 \\
0,8\end{array}$ & $\begin{array}{r}15,8 \\
16,7 \\
14,1 \\
6,9 \\
2,3\end{array}$ & $\begin{array}{r}13,5 \\
11,6 \\
0,8 \\
7,9 \\
1,9\end{array}$ \\
\hline
\end{tabular}


Roscoff [25]. Ils sont aussi comparables à ceux obtenus dans le système brassé profond du Georges Bank [15]. La forte utilisation d'ammoniun dans le système brassé profond est liée au maintien des concentrations élevées $(0,5-$ $\left.1,6 \mu \mathrm{mol} \mathrm{L} \mathrm{L}^{-1}\right)$ du début du printemps à la fin de l'été. Il est de plus reconnu que l'ammonium est la source d'azote utilisée préférentiellement par le phytoplancton [7]. Le nitrate est peu utilisé bien que sa concentration soit relativement élevée. Sa faible contribution à la nutrition azotée du phytoplancton peut résulter de l'effet inhibiteur de l'ammonium $[5,7,30,33]$; des concentrations de $0,5 \mu \mathrm{mol} \mathrm{L} \mathrm{L}^{-1}$ en ammonium sont en effet suffisantes pour réduire de plus de $50 \%$ l'utilisation du nitrate dans certaines régions côtières [16]. Elle doit également être liée aux conditions de lumière dans la colonne d'eau. En raison du brassage, les cellules phytoplanctoniques sont sans cesse déplacées à des niveaux de lumière différents et ne bénéficient pas, comme dans un système stratifié, d'un niveau de lumière constant dans la zone euphotique, mais d'une intensité moyenne d'éclairement dans la couche de mélange. Cette énergie lumineuse est réduite ; elle limite l'absorption de l'azote durant toute l'année [25], en particulier celle du nitrate, dont la dépendance vis-àvis de la lumière est plus marquée que celle de l'ammonium [26]. Le phytoplancton, dans l'ensemble du système brassé profond, utilise donc, quel que soit le secteur, l'ammonium et non le nitrate comme principal substrat azoté pour son développement.

\subsubsection{Système brassé peu profond}

En période de floraison printanière du phytoplancton (stations 4 et 6), les taux d'absorption des nitrates varient de 4,5 à $149 \mathrm{nmol} \mathrm{L}^{-1} \mathrm{~h}^{-1}$. Ceux de l'ammonium sont

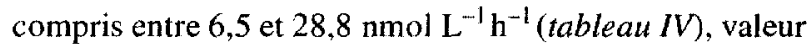
intégrée de l'absorption du nitrate atteint aux stations 4 et 6 respectivement 27,5 et $20,5 \mathrm{mmol} \mathrm{m}^{-2} \mathrm{j}^{-1}$; elle est trois fois supérieure à celle de l'ammonium (tableau $V$ ). Ces valeurs sont comparables à celles obtenues dans les eaux côtières brassées peu profondes de Loch Linnhe (Écosse) en période de développement printanier du phytoplancton

Tableau IV. Taux d'absorption du nitrate et de l'ammonium et taux de régénération de l'ammonium dans le système brassé peu profond.

Table IV. Uptake rates of nitrate and ammonium and regeneration rates of ammonium in the shallow well-mixed waters.

\begin{tabular}{|c|c|c|c|c|c|}
\hline & Stations & $\begin{array}{l}\text { Profondeur } \\
\text { (m) }\end{array}$ & $\begin{array}{c}\rho \mathrm{NO} \mathbf{O}_{3}^{-} \\
\left(\mathrm{nmol} \mathrm{L^{-1 }} \mathbf{h}^{-1}\right)\end{array}$ & $\begin{array}{c}\mathrm{\rho NH}_{4}^{+} \\
\left(\mathrm{nmol} \mathrm{L}^{-1} \mathbf{h}^{-1}\right)\end{array}$ & $\begin{array}{c}\mathbf{R N H}_{4}^{+} \\
\left(\mathrm{nmol} \mathrm{L}^{-1} \mathbf{h}^{-1}\right)\end{array}$ \\
\hline \multirow[t]{18}{*}{ Printemps } & Station 4 & 0 & 148,7 & 27,1 & 50,7 \\
\hline & & 3 & 108,9 & 27,1 & 19,0 \\
\hline & & 9 & 129,7 & 27,9 & 21,2 \\
\hline & & 15 & 63,4 & 28,8 & 16,1 \\
\hline & & 20 & 4,5 & 6,5 & 15,9 \\
\hline & & 25 & 4,5 & 7,7 & 16,6 \\
\hline & Station 6 & 0 & 87.7 & 17,6 & 20,6 \\
\hline & & 3 & 11,8 & 21,8 & 21,9 \\
\hline & & 7 & 108,4 & 20,1 & 7,6 \\
\hline & & 12 & 82,8 & 13,6 & 15,4 \\
\hline & & 16 & 67,7 & 21,9 & 9,6 \\
\hline & & 21 & 67,7 & 23,1 & 15,9 \\
\hline & Station 5 & 0 & 5,8 & 30.1 & 20,5 \\
\hline & & 5 & 6,3 & 34,9 & 29,7 \\
\hline & & 10 & 4,5 & 33,4 & 23,9 \\
\hline & & 15 & 7,0 & 24,1 & 12.2 \\
\hline & & 20 & 5,5 & 36,2 & 8,3 \\
\hline & & 30 & 1,8 & 36,2 & 23,3 \\
\hline \multirow[t]{5}{*}{ Été } & Station A & 0 & 9,7 & 32,5 & 20,8 \\
\hline & & 3 & 6,6 & 33,7 & 15,7 \\
\hline & & 9 & 3,8 & 28.7 & 16,5 \\
\hline & & 16 & 0,4 & 16,3 & 16,3 \\
\hline & & 38 & $<0,1$ & 13,7 & 13,7 \\
\hline
\end{tabular}


[35]. La forte utilisation du nitrate dans le système brassé peu profond, par rapport au système brassé profond, est liée aux meilleures conditions d'éclairement dont bénéficie le phytoplancton pour se développer. Dans le système brassé peu profond, une partie importante de la colonne d'eau est éclairée dès le printemps $\left(Z_{\text {mélange }} / Z_{\text {euphotique }}<1,5\right)$ favorisant l'absorption du nitrate. Après la floraison printanière (station 5), l'absorption du nitrate est inférieure à $7 \mathrm{nmol} \mathrm{L}^{-1} \mathrm{~h}^{-1}$ (tableau IV). Celle de l'ammonium atteint $36,2 \mathrm{nmol} \mathrm{L}^{-1} \mathrm{~h}^{-1}$. Intégrée sur la colonne d'eau, l'absorption de l'ammonium à la station 5 est environ six fois supérieure à celle du nitrate (tableau V). La nutrition azotée du phytoplancton cst donc nettement différente après la floraison printanière de celle observéc pendant la floraison elle-même. Ce changement de source d'azote est lié à l'épuisement du nitrate. L'absorption de l'ammonium se maintient après la floraison printanière, et c'est l'une des originalités de ce système, à des taux élevées. À la station $\mathrm{A}$, influencée par les apports de la Seine, l'absorption du nitrate est peu marquée malgré les fortes teneurs en ce composé. Celle de l'ammonium s'avère élevée (tableaux $I V, V$ ). La faible utilisation du nitrate doit correspondre à la présence d'ammonium à des concentrations relativement élevées et aux interactions entre ces deux formes d'azote (préférence et inhibition).

Ces résultats suggèrent que le système brassé peu profond de la Manche présente dans ses différents secteurs, un cycle de variations similaires d'absorption de l'azote. Les différences observées dans l'utilisation du nitrate et de l'ammonium pour une même période de l'annéc cor- respondent à un décalage dans la période de floraison printanière du phytoplancton. Dans les secteurs côtiers peu profonds, influencés par des apports anthropiques en azote, la colonne d'eau n'est pas épuisée en nitrate en été. Bien que ce composé domine le stock d'azote, l'ammonium est utilisé de manière prépondérante par le phytoplancton.

\subsection{Régénération de l'ammonium}

\subsubsection{Système brassé profond}

Au printemps, la régénćration de l'ammonium varic de 6,7 à $25,7 \mathrm{nmol} \mathrm{L}^{-1} \mathrm{~h}^{-1}$ dans le système brassé profond (tableau III). Intégrée sur la colonne d'eau, elle atteint respectivement 8,5 et $6,0 \mathrm{mmol} \mathrm{m}^{-2} \mathrm{j}^{-1}$ aux stations 1 et 3 . En été, des taux de régénération de 0,8 à $26,7 \mathrm{nmol} \mathrm{L}^{-1}$ $\mathrm{h}^{-1}$ ont été observés. Les valeurs intégrées sont comparables à celles mesurées au printemps (tableau $\mathrm{V}$ ).

\subsubsection{Système brassé peu profond}

Lors de la floraison printanière du phytoplancton, la régénération de l'ammonium dans le système brassé peu profond varie de 7,6 à $50,7 \mathrm{nmol} \mathrm{L} \mathrm{L}^{-1} \mathrm{~h}^{-1}$ (stations 4 et 6 , tableau $(V)$. Intégrée, elle atteint respectivement 7,2 et $6,7 \mathrm{mmol} \mathrm{m}^{-2} \mathrm{j}^{-1}$ aux stations 4 et 6 . Après la floraison printanière, des taux de régénération de 8.3 à $29,7 \mathrm{nmol} \mathrm{L}^{-1} \mathrm{~h}^{-1}$ sont mesurés à la station 5 (tableau $\mathrm{V}$ ). Ils représentent, intégrés sur la colonne d'eau, $10,9 \mathrm{mmol} \mathrm{m}^{-2} \mathrm{j}^{-1}$. À la station $\mathrm{A}$, les taux de régé-

Tableau V. Valeurs intégrées des taux d'absorption du nitrate et de l'ammonium, des taux de régénération de l'ammonium et du rapport $f$ dans le système brassế.

Table V. Depth-integrated values of nitrate and ammonium uptake rates, ammonium regeneration rates and f ratios in the well-mixed waters.

\begin{tabular}{|c|c|c|c|c|c|c|c|}
\hline & Stations & $\begin{array}{l}\text { Profondeur } \\
(\mathrm{m})\end{array}$ & 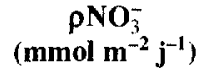 & $\underset{\left(\mathrm{mmol} \mathrm{m}^{-2} \mathrm{j}^{-1}\right)}{\mathrm{pNH}_{4}^{+}}$ & 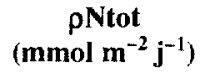 & $\begin{array}{c}\mathbf{R N H}_{4}^{+} \\
\left(\mathbf{m m o l ~ m}^{-2} \mathbf{j}^{-1}\right)\end{array}$ & rapport $f$ \\
\hline \multicolumn{8}{|c|}{ Système brassé profond } \\
\hline \multirow[t]{2}{*}{ Printemps } & Station 1 & 80 & 1,0 & 3,8 & 4.8 & 8,5 & 0,20 \\
\hline & Station 3 & 40 & 1,5 & 4.2 & 5,7 & 6,0 & 0,26 \\
\hline \multirow[t]{3}{*}{ Été } & Station $\mathrm{M}_{2}$ & 75 & 3,3 & 13.6 & 16,9 & 12,0 & 0,19 \\
\hline & Station $\mathrm{A}_{2}$ & 91 & 2,3 & 8,5 & 10,8 & 8,6 & 0,21 \\
\hline & Station $\mathrm{A}_{3}$ & 73 & 0,9 & 7,3 & 8,2 & 8,4 & 0,11 \\
\hline \multicolumn{8}{|c|}{ Système brassé peu profond } \\
\hline \multirow[t]{3}{*}{ Printemps } & Station 4 & 25 & 27,5 & 9,4 & 36,9 & 7.2 & 0,74 \\
\hline & Station 6 & 23 & 20,5 & 7,7 & 28,2 & 6.7 & 0,73 \\
\hline & Station 5 & 30 & 2,9 & 17,4 & 20,3 & 10,9 & 0,14 \\
\hline Été & Station A & 38 & 0,9 & 12,3 & 13.2 & 10.6 & 0,07 \\
\hline
\end{tabular}


nération d'ammonium sont compris entre 13,7 et $20,8 \mathrm{nmol} \mathrm{L}^{-1} \mathrm{~h}$ (tableau IV). La valeur intégrée est similaire à celle obtenue à la station 5 (tableau $V$ ).

Les taux de régénération de l'ammonium aux différentes stations étudiées sont comparables à ceux déjà relevés dans le système brassé profond [22] et peu profond [27] de la Manche occidentale. Nos résultats confirment l'intensité de la régénération de l'ammonium au printemps et en été dans l'ensemble du système brassé de la Manche. Intégrée sur la colonne d'eau, la régénération par les microhétérotrophes fournit, selon la station, de $62 \%$ à la totalité des besoins en ammonium du phytoplancton. Le recyclage de l'azote par ces microorganismes constitue donc une source d'ammonium importante pour le phytoplancton dans l'ensemble du système brassé. Il existe, à $50 \%$ de l'éclairement incident de surface, une relation linéaire $\left(r^{2}=0,83 ; n=9 ; p<0,01\right)$ entre les taux d'absorption et de régénération de l'ammonium (figure 3). Cette relation, établie à la profondeur correspondant au maximum de production, montre que l'activité phytoplanctonique est étroitement liée dans ce système à celle des microhétérotrophes.

\subsection{Production nouvelle} dans le système brassé de la Manche

La production nouvelle représente la production basée sur une utilisation d'azote importé dans la zone euphoti-

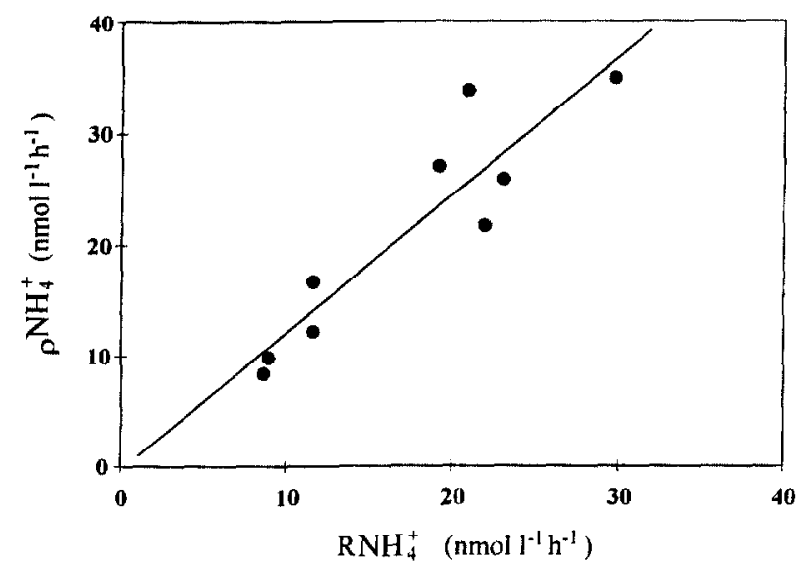

Figure 3. Variations des taux d'absorption de l'ammonium en fonction des taux de régénération de l'ammonium à $50 \%$ de la lumière incidente de surface $\left(\rho \mathrm{NH}_{4}^{+}=1,24 \mathrm{RNH}_{4}^{+}-0,38 ; p<0,01\right.$; $r^{2}=0,83$ ).

Figure 3. Relationship between uptake and regeneration rates of ammonium at $50 \%$ of light. que par des processus physiques, par opposition à la production régénérée résultant d'une utilisation d'azote recyclé in situ [8]. L'azote nouveau est essentiellement sous la forme de nitrate; la détermination de l'absorption du nitrate par le phytoplancton dans la zone euphotique permet donc d'estimer la production nouvelle. Les valeurs journalières de production nouvelle dans le système brassé profond sont faibles (tableau $V$ ) au regard de celles relevées sur les plateaux continentaux et la plupart des systèmes côtiers non perturbés par les apports anthropiques en azote $[6,31,36]$. Elles sont également pcu ćlevées dans le système brassé peu profond où de fortes valeurs sont observées uniquement durant la période de floraison printanière du phytoplancton. La part de la production nouvelle par rapport à la production totale (rapport $f,[10]$ ) est également faible dans le système brassé profond $(0,11-0,26)$ et dans le système brassé peu profond, après la floraison printanière $(0,07-0,14)$ (tableau V). Dans la plupart des systèmes côtiers, le rapport $f$ est compris entre 0,3 et 0,6 [32]. La production de nitrate par nitrification a été quantifiée au cours de cycles saisonniers dans les sytèmes brassés profond [21] et peu profond [29] de la Manche occidentale. Relativement faible lors de la floraison printanière, elle représente une source importante de nitrate pour le phytoplancton en été et fournit sur une base annuelle 25 à $35 \%$ du nitrate utilisé. La production basée sur l'utilisation de ce nitrate doit être considérée comme de la production régénérée. Les valeurs de production nouvelle er du rapport $f$ dans le système brassé de la Manche, estimées à partir de l'absorption du nitrate, correspondent donc à des valeurs maximales. Ces résultats soulignent l'importance du recyclage et de la production régénérée dans l'ensemble du système brassé de la Manche.

Les valeurs de production primaire en termes d'azote aux stations étudiées au printemps et en été (tableau $V$ ) sont relativement élevées. Elles se situent dans la gamme de valeurs habituellement relevées dans les systèmes côtiers non perturbés par les apports anthropiques en azote $[6,31,35]$. Ces fortes productions primaires ne résultent pas, tout au moins lorsque l'on ne prend pas en compte la période de floraison printanière du phytoplancton dans le système brassé peu profond, d'un apport d'azote nouveau pour l'écosystème, mais correspondent à de fortes productions régénérées. Cette situation différencie nettement l'ensemble du système brassé de la Manche de la plupart des systèmes côtiers, où à de fortes productions azotées correspondent habituellement des productions nouvelles élevées $[10,16]$. L'importance de la production 
régénérée dans le système brassé doit être liée au recyclage in situ de l'azote par les microhétérotrophes. Elle est associée au régime de mélange vertical des masses d'eaux. En empêchant l'installation de la thermocline, le brassage maintient le système à une couche toute l'année. La matière végétale produite dans la zone euphotique se répartil sur toute la colonne d'eau et les produits issus de la minéralisation de cette matière organique restent disponibles pour la production primaire. Dans un système stratifié, la situation est différente. Une fraction importante de la matière organique sédimente sous la thermocline [17], l'azote régénéré dans la couche de fond devient alors indisponible pour unc large part pour la production primaire. Le brassage permet un renouvellement continu de l'azote dans la zone euphotique et entretient une régénération active sur une longue période. La quantité d'azote disponible pour la production primaire, puis par la suite pour la régénération, est donc importante. Il en résulte, dans l'ensemble du système brassé de la Manche, une production régénérée importante et une production primaire relativement élevée.

\section{CONCLUSIONS}

Le système brassé profond présente, dans les différents secteurs de la Manche, des caractéristiques similaires : le nitrate est peu utilisé et l'absorption de l'ammonium est élevée dès le printemps et durant toute la période estivale. Dans le système brassé peu profond, l'absorption du nitrate est élevée uniquement lors de la floraison du phytoplancton. Celle de l'ammonium s'avère importante dès la fin de la floraison printanière et en période estivale. Elle représente alors la quasi-totalité de l'azote utilisé. La forte utilisation d'ammonium dans l'ensemble du système est liée à un recyclage rapide de l'azote dans la colonne d'eau. La production primaire est élevée. Elle résulte dans le système brassé profond d'une production régénérée importante. La situation est similaire dans le système brassé peu profond en dehors de la période de floraison printanière du phytoplancton.

\section{Remerciements}

Ce travail est une collaboration du Programme national d'océanographie côtière (PNOC).

\section{RÉFÉRENCES}

[1] Birrien J.L., Wafar M.V.M., Le Corre P., Riso R., Nutrient and primary production in a shallow stratified ecosystem in the Iroise Sea, J. Plankt. Res. 13 (1991) 721-742.

[2] Boalch G.T., Harbour D.S., Butler E.L., Seasonal phytoplankton in the Western English Channel 1964-1974, J. Mar. Biol. Ass. U.K. 58 (1978) 953-953.

[3] Brunet C., Brylinski J.M., Bodineau L., Thoumelin G., Bentley D., Hilde D., Phytoplankton dynamics during the spring bloom in the south eastern English Channel, Estuar. Coast. Shelf Sci. 43 (1996) 469-483.

[4] Brylinski J.M., Lagadeuc Y., Gentilhomme V., Le fleuve côtier: un phénomène hydrologique important en Manche orientale, exemple du Pas de Calais, Oceanol. Acta 11 (1991) 197-203.

[5] Carpenter E.J., Dunham S., Nitrogenous nutrient uptake, primary production and species composition of phytoplankton in the Carmans River estuary, Long Island, New York, Limnol. Oceanogr. 30 (1985) 513-526.

[6] Cochlan W.P., Seasonal study of uptake and regeneration of nitrogen on the Scotian Shelf, Cont. Shelf Res. 5 (1986) 555 577.

[7] Dortch Q., The interaction between ammonium and nitrate uptake in phytoplankton, Mar. Ecol. Prog. Ser. 61 (1990) $183-$ 201.
[8] Dugdale R.C., Goering J.J., Uptake of new and regenerated forms of nitrogen in primary productivity, Limnol. Oceanogr. 12 (1967) 685-695.

[9] Dugdale R.C., Wilkerson F.P., The use of ${ }^{15} \mathrm{~N}$ to measure nitrogen uptake in eutrophic oceans; experimental conditions, Limnol. Oceanogr. 31 (1986) 673-689.

[10] Eppley R.W., Peterson B.J., Particulate organic matter flux and planktonic new production in the deep ocean, Nature 282 (1979) 677-680.

[1I] Glibert P.M., Lipchultz F., McCarthy J.J., Altabet M.A., Isotope dilution models of uptake and remineralization of ammonium by marine plankton, Limnol. Oceanogr. 27 (1982) 639-650.

[12] Grall J.R., Recherches quantitatives sur la production primaire du phytoplancton dans les parages de Roscoff, thèse de doctorat ès sciences naturelles, université de Paris-VI, France (1972) $259 \mathrm{p}$.

[13] Guiraud D., Fardeau J.C., Détermination isotopique par spectrométrie optique de composés faiblement enrichis en azote15. Analusis 8 (1980) 148-152.

[14] Harrison W.G., Experimental measurements of nitrogen remineralization in coastal waters, Limnol. Oceanogr. 23 (1978) 684-694.

[15] Harrison W.G., Douglas D., Falkowski P., Rowe G., Vidal J., Summer nutrient dynamics of the Middle Atlantic Bight: 
nitrogen uptake and regeneration, J. Plankt. Res. 5 (1983) 539-556.

[16] Harrison W.G., Platt T., Lewis M.R., $f$-ratio and its relationship to ambient nitrate concentration in coastal waters, J. Plankt. Res. 9 (1987) 235--248.

[17] Heiskanen A.S., Leppanen J.M., Estimation of export production in the coastal Baltic Sea: effect of resuspension and microbial decomposition on sedimentation measurements, Hydrobiologia 316 (1995) 211-224.

[18] Kanda J., Ziemman D.A., Conquest L.D., Bienfang P.K., Nitrate and ammonium uptake by phytoplankton over the spring bloom in Auke Bay, Alaska, Estuar. Coast. Shelf Sci. 30 (1990) 509-524.

[19] Koroleff F., Direct deternination of anmonia in natura] wale]s as indophenol blue, in: Information on techniques and methods for seawater, Rapp. P.V. Réun. Cons. Perm. Int. Explor. Mer 3 (1970) 19-22.

[20] Kristiansen S., Paasche E., An improved method for determining relative ${ }^{15} \mathrm{~N}$ abundance in ammonium regeneration studies by direct diffusion, Mar. Ecol. Prog. Ser. 54 (1989) 203-207.

[21] Laws E.A., Isotope dilution models and the mystery of the vanishing ${ }^{15} \mathrm{~N}$, Limnol. Oceanogr. 29 (1984) 379-386.

[22] Le Corre P., Wafar M.V.M., L'Helguen S., Maguer J.F., Ammonium assimilation and regeneration by fractionated plankton in permanently well-mixed temperate waters, J. Plankt. Res, 18 (1996) 355-370.

[23] L'Helguen S., Absorption et régénération de l'azote dans les écosystèmes pélagiques du plateau continental de la Manche occidentale, relations avec le régime de mélange vertical des masses d'eaux, cas du front thermique d'Ouessant, thèse de doctorat de spécialité, chimie appliquéc: chimic marinc, université de Bretagne occidentale, Brest (1991) 212 p.

[24] L'Helguen S., Madec C., Le Corre P., Nutrition azotée du phytoplancton dans les eaux brassées de la Manche occidentale, Oceanol. Acta 16 (1993) 653-660.

[25] L'Helguen S., Madec C., Le Corre P., Nitrogen uptake in permanently well-mixed temperate coastal waters, Estuar. Coast. Shelf Sci. 42 (1996) 803-818.

[26] MacIsaac J.J., Dugdale R.C., Interactions of light and inorganic nitrogen in controlling nitrogen uptake in the sea, DeepSea Res. 16 (1972) 45-57.

[27] Maguer J.F., Absorption el régénération de l'azote dans les écosystèmes côtiers, relation avec le régime de mélange vertical des masses d'eaux, cas du système homogène peu profond de la Manche occidentale, thèse de doctorat de spécialité, chimie appliquée : chimie marine, université de Bretagne occidentale, Brest (1995) 194 p.

[28] Maguer J.F., Le Corre P., Madec C., Variations saisonnières de l'absorption de l'azote dans un système brassé peu profond (Manche occidentale), Oceanol. Acta 19 (1996a) 529-537.

[29] Maguer J.F., Le Corre P., L'Helguen S., Sources d'azote pour le phytoplancton dans le système brassé peu profond de la Manche occidentale, importance de la production régénérée, bilan annuel, J. Rech. Océanogr. 21 (1996b) 103-108.

[30] McCarthy J.J., Taylor W.R., Taft J.L., Nitrogenous nutrition of the plankton in the Chesapeake Bay, 1: Nutrient availability and phytoplankton preferences, Limnol. Oceanogr. 22 (1977) 996-1011.

[31] Owens N.J.P., Mantoura R.C.F., Burkill P.H., Howland R.J.M., Pomroy A.J., Woodward E.M.S., Nutrient cycling studies in Carmarthen Bay: phytoplankton production, nitrogen assimilation and regeneration, Mar. Biol. 93 (1986) 329-342.

[32] Paasche E., Pelagic primary production in nearshore waters, in: Nitrogen cycling in coastal marine environments, Blackburn T.H., Sorensen J., (Eds), John Wileys and Sons (1988) 33-57.

[33] Paasche E., Kristiansen S., Nitrogen nutrition of the phytoplankton in the Oslofjord, Estuar. Coast. Shelf Sci. 14 (1982) 237-249.

[34] Pettersson K. Seasonal uptake of carbon and nitrogen and intracellular storage of nitrate in planktonic organisms in the Skagerrak, J. Exp. Mar. Biol. Ecol. 151 (1991) 121-137.

[35] Rees A.P., Owens N.J.P., Heah M.R., Plunner D.H., Bellerby R.S., Seasonal nitrogen assimilation and carbon fixation in a fjordic sea loch. J. Plankt. Res. 17 (1995) 1307-1325.

[36] Sahlsten S., Sorenssön F., Petterson K., Planktonic nitrogen uptake in the south eastern Kattegat. J. Exp. Mar. Biol. Ecol,, 121 (1988) 227-246.

[37] Tréguer P., Le Corre P., Manuel d'analyse des seis nutritifs dans l'eau de mer (utilisation de l'autoanalyseur II. Technicon), $2^{\mathrm{e}}$ éd., université de Bretagne occidentale, Brest, France, $1975,110 \mathrm{p}$.

[38] Wafar M.V.M.. Le Corre P., Birrien J.L., Nutrients and primary production in permanently well-mixed temperate coastal waters, Estuar. Coast. Shelf Sci. 17 (1983) 431-446.

[39] Yentsch C.S., Menzel D.W., A method for the determination of phytoplankton chlorophyll and phaeophytin by fluorescence, Deep-Sea Res. 10 (1963) 221-231. 